\title{
A Challenging Case of Refractory Biliary Leak in a Patient With Hydatid Liver Disease
}

\author{
Aws Alameri ${ }^{\mathrm{a}, \mathrm{e}}$, Mohammed Alkhero ${ }^{\mathrm{b}}$, Alfarooq Alshaikhlic ${ }^{\mathrm{c}}$, Abbas Alshami ${ }^{\mathrm{d}}$, James Saca ${ }^{\mathrm{a}}$
}

\begin{abstract}
Hydatid liver disease (HLD) is the most common form of hydatid disease, and it is caused by a zoonotic infection with a tape worm. It is endemic mostly in sheep-farming countries and rare in the United States. Liver involvement is usually asymptomatic, but symptoms develop upon growth of the cyst leading to many complications, most common of which is intra-biliary rupture, and less likely biliary obstruction. Diagnosis is clinical, serologic and radiologic. Therapeutic approaches to HLD include surgery, anthelminthic medications and medico-surgical procedures. Here we present a case of HLD that presented in advanced stage leading to grave consequences, complicated course and difficult therapeutic options. Given the rarity of hydatid disease in Northern America, physicians have to keep high index of suspicion especially in a patient with history of travel to endemic areas, as early diagnosis and treatment is important to avoid high morbidity and mortality.
\end{abstract}

Keywords: Hydatid disease; Intra-biliary rupture; Cystobiliary fistula; Refractory hydatid liver disease

\section{Introduction}

Hydatid disease (HD) is a zoonotic parasitic infection caused by tape worm species Echinococcus granulosus or to a lesser extent Echinococcus multilocularis causing cystic or alveolar echinococcosis respectively. The tape worm of Echinococcus granulosus is found in the small bowel of carnivores,

Manuscript submitted March 10, 2021, accepted March 24, 2021

Published online May 13, 2021

a Department of Internal Medicine, University of Texas Health Science Center at San Antonio, 7703 Floyd Curl Drive, San Antonio, TX 78229-3900, USA bDepartment of Internal Medicine, UHS Southern California Medical Education Consortium, 42211 Stonewood Rd, Temecula, CA 92591, USA

'Department of Internal Medicine, University of Texas, Rio Grande Valley at DHR, 5423 S McColl Rd, Edinburg, TX 78539, USA

dDepartment of Internal Medicine, Jersey Shore University Medical Center, 1945 NJ-33, Neptune City, NJ 07753, USA

${ }^{e}$ Corresponding Author: Aws Alameri, Department of Internal Medicine, University of Texas Health Science Center at San Antonio, 7703 Floyd Curl Drive, San Antonio, TX 78229-3900, USA. Email: alameria@uthscsa.edu

doi: https://doi.org/10.14740/jmc3694 mainly dogs, and its eggs get ingested by intermediate hosts that involve humans and animals like sheep, cattle, goats and pigs [1-3]. It is more common in sheep-rearing areas like Asia mainly in the Mediterranean countries, Australia, Europe, Africa, Central and South America, and in rare cases in North America [1, 2]. In the United States, it is more endemic in certain regions in Utah, California and Alaska and immigration has increased the number of cases outside endemic areas [4]. The most common site of involvement is the liver that is affected in $70-80 \%$ of cases, followed by the lung that is involved in about $15-25 \%$ of patients, and involvement of other organs is very rare [1, 5-7]. Lines of treatment include surgery, medical therapy and percutaneous aspiration. We present an advanced case of hydatid liver disease (HLD) in a low prevalence area that was complicated by intra-biliary rupture (IBR) and refractory biliary leak to usual lines of management.

\section{Case Report}

A 52-year-old woman with a known liver cyst and gall stone disease presented with 1 week of right upper quadrant (RUQ) pain, nausea without vomiting, anorexia, jaundice and itching. She presented similarly 6 years before. At that time, computed tomography (CT) scan was suspicious for hydatid cyst, and local hospital echinococcal antibodies were positive. However, sent-out repeat tests were negative. The patient denied contact with animals but did have multiple visits to Jerusalem. Given low epidemiology in the United States, negative repeat tests and low suspicion, the patient was scheduled to undergo hepatic cyst resection for diagnosis, but she was lost to follow-up. On this admission, the patient was afebrile and had scleral icterus with mildly jaundiced skin. Laboratory results revealed eosinophilia (white blood cell count of $7,200 / \mu \mathrm{L}$ with absolute eosinophil count of $710 / \mu \mathrm{L}, \mathrm{Hb}$ of $14 \mathrm{~g} / \mathrm{dL}$ and platelet count of $299,000 / \mu \mathrm{L}$ ) with biliary pattern liver function tests that showed alkaline phosphatase (ALP) of $367 \mathrm{U} / \mathrm{L}$ with total bilirubin of $3.5 \mathrm{mg} / \mathrm{dL}$ and direct bilirubin of 3.0 $\mathrm{mg} / \mathrm{dL}$ with elevated transaminases (aspartate transaminase (AST) $136 \mathrm{U} / \mathrm{L}$ and alanine aminotransferase (ALT) $244 \mathrm{U} / \mathrm{L}$ ). This time, echinococcal antibodies were positive for both local and sent-out ones. Ultrasound showed cholelithiasis without evidence of cholecystitis and calcified right hepatic cystic lesion. CT scan demonstrated large hepatic hypodense lesion with thick calcified rim (Fig. 1). Magnetic resonance cholangiopancreatography (MRCP) showed hepatic cyst along with 


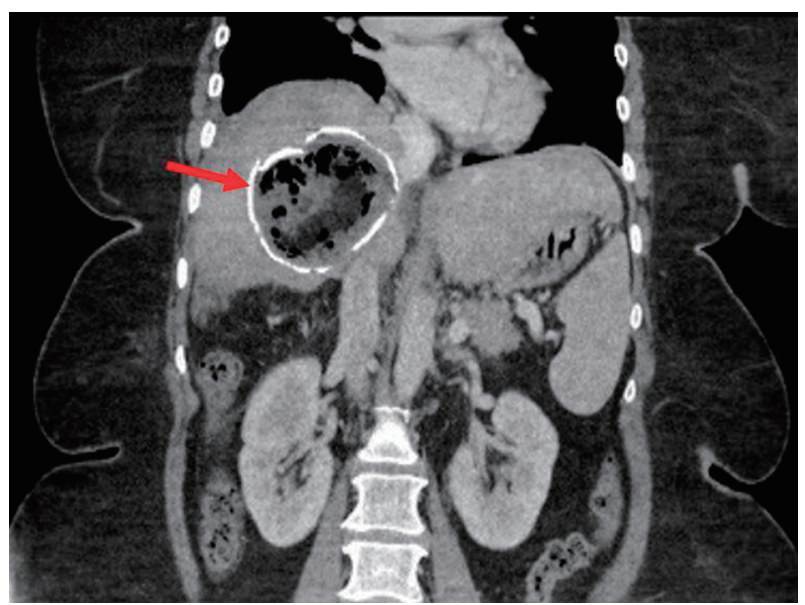

Figure 1. Coronal section view of computed tomography scan showing minimally complex cyst of the right liver lobe with thick rim of calcification (arrow).

dilated central bile ducts and abrupt narrowing at the biliary confluence (Fig. 2). The endoscopic retrograde cholangiopancreatography (ERCP) revealed mild stenosis in the upper third of the common bile duct (CBD) from external compression, and the copious mucus obtained through balloon sweeps showed scolices. Based on the presentation and workup, the patient was diagnosed with HLD concerning for cystobiliary fistula and was started on albendazole. The patient was scheduled for cholecystectomy and surgical cyst removal. Since the cyst was densely adherent to the right hepatic vein and inferior vena cava, radical resection was impractical. Therefore, she underwent partial cystectomy, cystobiliary fistula repair and omentoplasty. Subsequent intraoperative cholangiogram

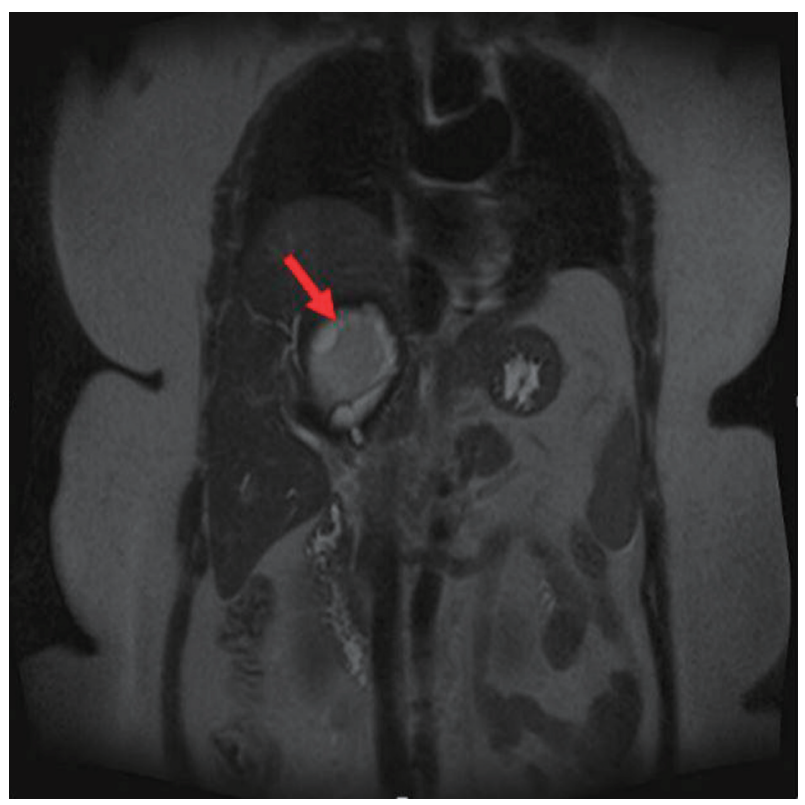

Figure 2. Coronal section of magnetic resonance cholangiopancreatography showing minimally complex right hepatic cyst with rim of calcification and proteinaceous or hemorrhagic contents (arrow).

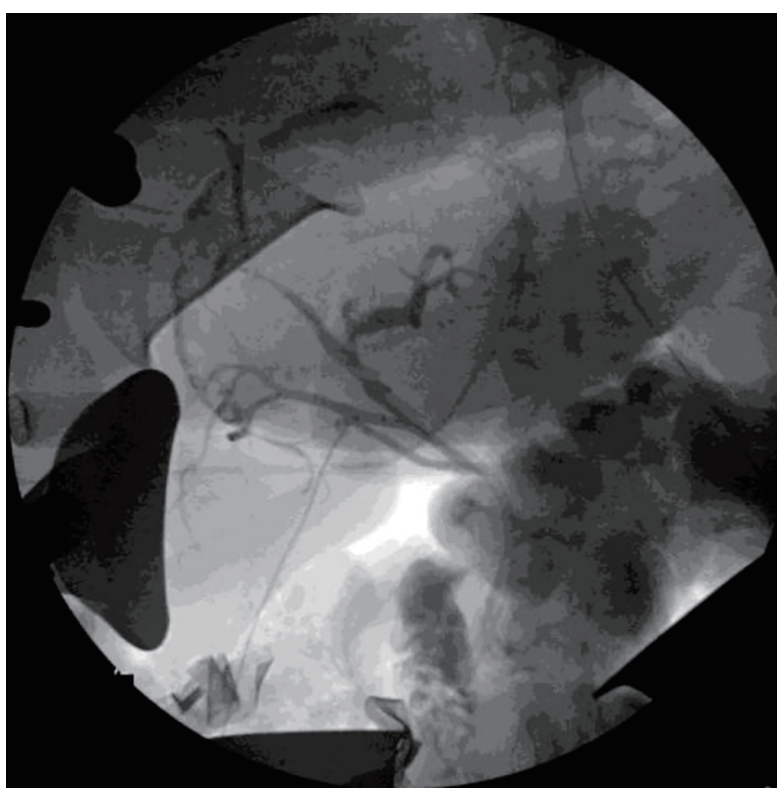

Figure 3. Intraoperative cholangiogram showing no evidence of intraoperative biliary leak.

showed no leak (Fig. 3). However, 2 weeks after the surgery, the patient developed a refractory biliary leak for which aggressive endotherapy, necrosectomy procedures and cystobiliary fistula embolization failed (Fig. 4). The case was further complicated by hepatic abscess and colonic fistula that required multiple admissions and variable antibiotic courses, and for which the patient is planned to undergo percutaneous closure. Probable next step, per multidisciplinary team discussions, is right hepatectomy with or without Roux-en-Y hepaticojejunostomy, and right hemicolectomy with diverting loop ileostomy.

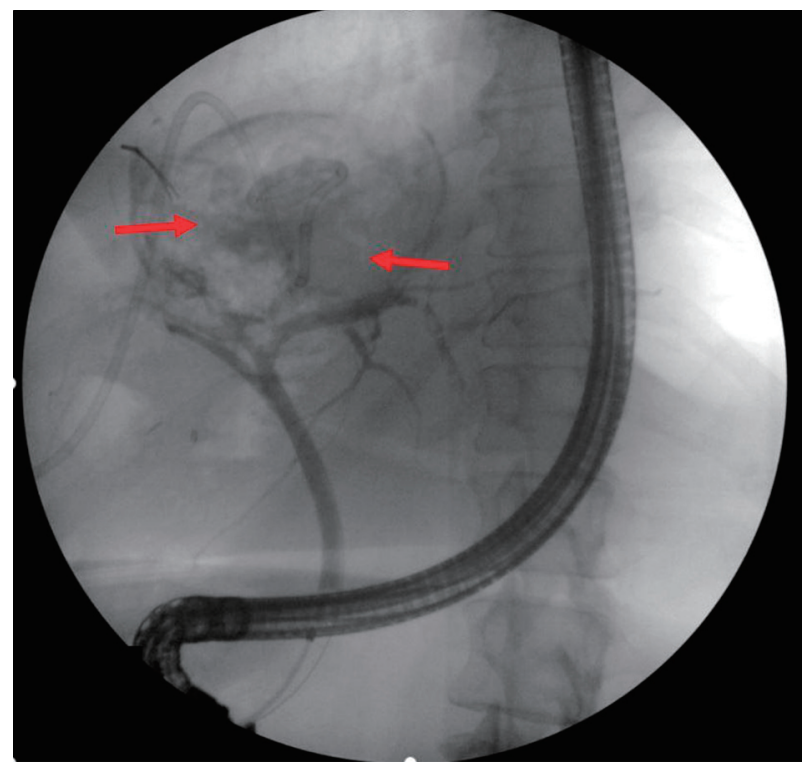

Figure 4. Postoperative endoscopic retrograde cholangiopancreatography showing right and left hepatic ducts leakage (arrows). 


\section{Discussion}

HLD is the most common form of HD and is usually asymptomatic at variable length and then starts to develop symptoms secondary to complications like infection, biliary obstruction or rupture into biliary tree $[1,4]$. The most common and serious complication is IBR that occurs in about $2-42 \%$ of cases. It is mostly occult due to small tears and communication between the cyst and the biliary radicals or less likely frank rupture involving large biliary ducts. Rupture occurs mostly centrally and is precipitated by physical trauma, and excessive coughing or vomiting [7-9]. Dew was first who described IBR and mentioned that rupture mostly occurs in the biliary ducts of the right liver lobe followed by those of the left liver lobe and rarely in the CBD $[8,10]$. The rupture can be contained within the pericyst, communicating with biliary tree or direct rupture into the peritoneal cavity according to Lewall and McCorkell [7, 11]. This can lead to obstructive jaundice, cholecystitis, cholangitis, pancreatitis, hepatic abscess, sepsis, dissemination and anaphylaxis $[1,4,8,12]$. To a lesser extent, HLD can be complicated by growth of the cyst and pressure on hepatic parenchyma or causing biliary obstruction [1, 7].

The diagnosis of HD requires combination of clinical feature, serology and imaging [13]. Serologic tests that use the detection of hydatid antigen are employed to help in diagnosis, most common of which is enzyme-linked immunosorbent assay (ELISA) [14]. First line of radiologic diagnosis is ultrasonography (USG). On USG, Gharbi et al described five types of echinococcal cysts and WHO described international classification in 2003 that those are believed to correspond to the evolution stage of hydatid cyst $[7,13,15,16]$. While CT scan is not essential for diagnosing HLD but gives higher definition in terms of cyst number, size, content and calcification [12]. Moreover, it is useful in diagnosing biliary hydatid disease (BHD) and in preoperative planning [7]. Similarly, magnetic resonance imaging (MRI) helps in diagnosis of HLD and BHD whenever CT scan and USG are inconclusive $[7,17]$. MRCP is another diagnostic tool to help confirm IBR cases [7].

ERCP has been successfully used diagnostically or therapeutically in managing HLD and HLD-related complications $[1,4]$. Although the routine preoperative use of ERCP in an uncomplicated HD is not accepted yet, some centers advise its use to define the anatomy of biliary tree and evaluate for any cysto-biliary communication and thence decrease postoperative complications [8]. ERCP has been considered the mainstay in evaluating biliary communications as the direct visualization of the communication between the cyst and the biliary radicals is the most reliable method [9]. However, in about $0-30 \%$ of patients with suspected frank IBR (i.e., presented with jaundice and/or dilated bile ducts) and who underwent ERCP, only external bile duct compression by the cyst was observed [8]. Furthermore, minor cysto-biliary communications can also be missed in ERCP due to increased cyst pressure, daughter cyst, or old hydatid material that can block contrast from demonstrating the communication [8].

Regarding the classical surgical treatment, mortality is around $0.9-3.6 \%$ and the recurrence rate is around $11.3 \%$ in the first 5 years [18]. The classical surgery procedures used for the treatment of the hepatic hydatid cyst are divided, according to their attitude towards the pericyst, into procedures that do not involve pericyst resection (cystectomy) and procedures involving pericyst resection (partial pericystectomy, pericystoresection and hepatectomy). They are associated with procedures that should treat the remaining cavity: external drainage with a drain tube, bipolar drainage of the cavity and the main bile duct, padding, omental plombage, drainage of the cavity by anastomosis with the stomach/jejunum and pericysto-biliar drainage. It should also be mentioned that hepatic transplantation might be a treatment option when at least $25-30 \%$ of the total hepatic parenchymal volume cannot be saved, or in the case of para- or post-hydatid hepatic cirrhosis [19].

\section{Acknowledgments}

None to declare.

\section{Financial Disclosure}

None to declare.

\section{Conflict of Interest}

None to declare.

\section{Informed Consent}

Verbal consent was obtained and patient data were de-identified.

\section{Author Contributions}

Aws Alameri obtained the case. All authors contributed to the writing, reviewing and final approval of the case.

\section{Data Availability}

The authors declare that data supporting the findings of this study are available within the article.

\section{References}

1. Simsek H, Ozaslan E, Sayek I, Savas C, Abbasoglu O, Soylu AR, Balaban Y, et al. Diagnostic and therapeutic ERCP in hepatic hydatid disease. Gastrointest Endosc. 2003;58(3):384-389.

2. Guidelines for treatment of cystic and alveolar echinococcosis in humans. WHO Informal Working Group on Echinococcosis. Bull World Health Organ. 1996;74(3):231-242.

3. Prevention C-C for DC and CDC - Echinococcosis - Epi- 
demiology \& Risk Factors. Published April 16, 2019. Accessed May 3, 2020. https://www.cdc.gov/parasites/ echinococcosis/epi.html.

4. Bristow BN, Lee S, Shafir S, Sorvillo F. Human echinococcosis mortality in the United States, 1990-2007. PLoS Neg1 Trop Dis. 2012;6(2):e1524.

5. Krasniqi A, Bicaj B, Limani D, Maxhuni M, Rrusta A, Hoxha F, Hamza A, et al. The role of perioperative endoscopic retrograde cholangiopancreatography and biliary drainage in large liver hydatid cysts. ScientificWorldJournal. 2014;2014:301891.

6. Safioleas M, Stamoulis I, Theocharis S, Moulakakis K, Makris S, Kostakis A. Primary hydatid disease of the gallbladder: a rare clinical entity. J Hepatobiliary Pancreat Surg. 2004;11(5):352-356.

7. Somani SK. Resolution of hepatic hydatid cyst with biliary communication with ERCP. J Gastrointest Digest Sys. 2012;02(04):114.

8. Dolay K, Akbulut S. Role of endoscopic retrograde cholangiopancreatography in the management of hepatic hydatid disease. World J Gastroenterol. 2014;20(41):1525315261.

9. Gupta P, Debi U, Sinha SK, Prasad KK. Role of endoscopic retrograde cholangiography in ruptured hepatic hydatid cyst. Trop Gastroenterol. 2015;36(1):21-24.

10. Dew H. Some complications of hydatid disease. Br J Surg. 1930;18(70):275-293.

11. Lewall DB, McCorkell SJ. Rupture of echinococcal cysts: diagnosis, classification, and clinical implications. AJR Am J Roentgenol. 1986;146(2):391-394.

12. Jourdan J-L, Morris DL. Hydatid liver disease. Zuckschwerdt. 2001. Accessed May 4, 2020. http://www.ncbi. nlm.nih.gov/books/NBK7009/.

13. Mantonakis E, Papalampros A, Moris D, Dimitrokallis N, Sakarellos P, Griniatsos J, Felekouras E. Radiofrequency energy in hepatic bed during partial cystectomy for hydatid liver disease: standing out from the usual conservative surgical management. Gastroenterol Res Pract. 2016;2016:1078653.

14. Parija SC. A review of some simple immunoassays in the serodiagnosis of cystic hydatid disease. Acta Trop. 1998;70(1):17-24.

15. Gharbi HA, Hassine W, Brauner MW, Dupuch K. Ultrasound examination of the hydatic liver. Radiology. 1981;139(2):459-463.

16. WHO Informal Working Group. International classification of ultrasound images in cystic echinococcosis for application in clinical and field epidemiological settings. Acta Trop. 2003;85(2):253-261.

17. Kumar R, Reddy SN, Thulkar S. Intrabiliary rupture of hydatid cyst: diagnosis with MRI and hepatobiliary isotope study. Br J Radiol. 2002;75(891):271-274.

18. Derbel F, ed. Abdominal surgery. BoD - Books on Demand, 2012.

19. Bratucu E. Manual de chirurgie pentru studenti. Editura Universitara "Carol Davila". 2009. 